\title{
DIGITALCOMMONS
}

$11-1-2012$

\section{End Matter}

JMASM Editors

Follow this and additional works at: http:// digitalcommons.wayne.edu/jmasm

\section{Recommended Citation}

Editors, JMASM (2012) "End Matter," Journal of Modern Applied Statistical Methods: Vol. 11 : Iss. 2 , Article 25. DOI: $10.22237 /$ jmasm $/ 1351743840$

Available at: http://digitalcommons.wayne.edu/jmasm/vol11/iss2/25 


\section{Instructions For Authors}

Follow these guidelines when submitting a manuscript:

1. JMASM uses a modified American Psychological Association style guideline.

2. Submissions are accepted via e-mail only. Send them to the Editorial Assistant at ea@jmasm.com. Provide name, affiliation, address, e-mail address, and 30 word biographical statements for all authors in the body of the email message.

3. There should be no material identifying authorship except on the title page. A statement should be included in the body of the e-mail that, where applicable, indicating proper human subjects protocols were followed, including informed consent. A statement should be included in the body of the e-mail indicating the manuscript is not under consideration at another journal.

4. Provide the manuscript as an external e-mail attachment in MS Word for the PC format only. (Wordperfect and .rtf formats may be acceptable - please inquire.) Please note that Tex (in its various versions), Exp, and Adobe .pdf formats are designed to produce the final presentation of text. They are not amenable to the editing process, and are NOT acceptable for manuscript submission.

5. The text maximum is 20 pages double spaced, not including tables, figures, graphs, and references. Use 11 point Times New Roman font.

6. Create tables without boxes or vertical lines. Place tables, figures, and graphs "in-line", not at the end of the manuscript. Figures may be in .jpg, tif, .png, and other formats readable by Adobe Photoshop.

7. The manuscript should contain an Abstract with a 50 word maximum, following by a list of key words or phrases. Major headings are Introduction, Methodology, Results, Conclusion, and References. Center headings. Subheadings are left justified; capitalize only the first letter of each word. Sub-subheadings are left-justified, indent optional. Do not number headings or subheadings.

8. Number all formulas, tables, figures, and graphs, but do not use italics, bold, or underline.

9. Do not use underlining in the manuscript. Do not use bold, except for (a) matrices, or (b) emphasis within a table, figure, or graph. Do not number references. Do not use footnotes or endnotes.

10. In the References section, do not put quotation marks around titles of articles or books. Capitalize only the first letter of books. Italicize journal or book titles, and volume numbers. Use " $\&$ " instead of "and" in multiple author listings.

11. Suggestions for style: Instead of "I drew a sample of 40 " write "A sample of 40 was selected". Use "although" instead of "while", unless the meaning is "at the same time". Use "because" instead of "since", unless the meaning is "after". Instead of "Smith (1990) notes" write "Smith (1990) noted". Do not strike spacebar twice after a period.

\section{Print Subscriptions}

Print subscriptions including postage for professionals are US \$95 per year; for graduate students are US $\$ 47.50$ per year; and for libraries, universities and corporations are US \$195 per year. Subscribers outside of the US and Canada pay a US \$10 surcharge for additional postage. Online access is currently free at http://www.jmasm.com/. Mail subscription requests with remittances to JMASM, P. O. Box 48023, Oak Park, MI, 48237. Email journal correspondence, other than manuscript submissions, to ea@jmasm.com.

\section{Notice To Advertisers}

Send requests for advertising information to ea@jmasm.com. 